\title{
Qual droga utilizar para o tratamento prolongado de tromboembolismo venoso?
}

\author{
Which drug to use for extended treatment of venous thromboembolism?
}

Andressa Zacharias Bendine (®), José Augusto Costa (†

O tromboembolismo venoso (TEV) é a terceira causa mais comum de morte vascular. $\mathrm{O}$ tratamento principal é a anticoagulação, e, nos pacientes sem câncer ativo, as diretrizes sugerem uso de anticoagulantes orais, como rivaroxabana, a antagonistas da vitamina $\mathrm{K}$, como a varfarina.

A terapia anticoagulante é administrada por três meses ou mais, dependendo do risco de recorrência de TEV versus risco de sangramento.

Nos pacientes sem fatores de risco reversíveis, o risco de recorrência é de $10 \%$ no primeiro ano se o tratamento é cessado.

Apesar da terapia de anticoagulação prolongada ser efetiva para prevenir o TEV recorrente, a preocupação com o sangramento muitas vezes leva a uma insegurança no tratamento anticoagulante por mais de 6 ou 12 meses.

Tentativas de reduzir o risco de sangramento quando o tratamento é prolongado incluem o uso de doses menores de anticoagulantes ou de aspirina como substituto.

A rivaroxabana foi efetiva para a prevenção de acidente vascular encefálico (AVE) em pacientes com fibrilação atrial, para o tratamento e na profilaxia de TEV, após artroplastias de joelho ou quadril.

No estudo "Einstein Choice" (Bayer), ${ }^{1}$ os autores compararam a eficácia e a segurança das duas doses de rivaroxabana (10 e $20 \mathrm{mg}$ ) com $100 \mathrm{mg}$ de aspirina em pacientes com TEV que completaram 6 e 12 meses de tratamento anticoagulante randomizados em três grupos (rivaroxabana $10 \mathrm{mg} / \mathrm{dia}$, rivaroxabana $20 \mathrm{mg} /$ dia e aspirina $10 \mathrm{mg} / \mathrm{dia}$ ).

Objetivos secundários do estudo foram determinar se a dose menor de rivaroxabana foi tão efetiva quanto a dose maior e se foi associada com sangramento menor.

Os desfechos negativos foram TEV recorrente não fatal, fatal e mortes inexplicadas em que o tromboembolismo pulmonar não pode ser excluído.

O principal desfecho de segurança foi sangramentos maiores. Outros desfechos negativos de segurança foram sangramen- tos menores clinicamente relevantes que levaram à interrupção do estudo da droga por mais de 14 dias.

O seguimento do estudo realizou-se com avaliações periódicas de 30, 90, 180, 270 e 360 dias dos pacientes e após 30 dias de interrupção do medicamento. Pacientes foram orientados a procurar o centro de estudo caso apresentassem recorrência dos sintomas ou sangramento. Em dois anos, contabilizou-se um total de 3.365 pacientes de 244 centros em 31 países para análise primária.

O sangramento maior ocorreu em seis pacientes no grupo de $20 \mathrm{mg}$ de rivaroxabana $(\mathrm{n}=1.107)$, cinco no grupo de $10 \mathrm{mg}$ de rivaroxabana $(n=1.127)$ e três no grupo da aspirina $(n=1.131)$.

Infarto agudo do miocárdio, acidente vascular cerebral ou embolia sistêmica ocorreram em três pacientes no grupo de 20 $\mathrm{mg}$ de rivaroxabana $(0,3 \%)$, em cinco $(0,4 \%)$ no grupo de $10 \mathrm{mg}$ de rivaroxabana e em sete $(0,6 \%)$ no grupo aspirina.

As taxas de mortalidade de qualquer causa foram de $0,7 \mathrm{e}$ $0,2 \%$ no grupo de $20 \mathrm{mg}$ e $10 \mathrm{mg}$ de rivaroxabana, respectivamente, comparado com $0,6 \%$ do grupo da aspirina. Estudos prévios mostraram que, em comparação com o placebo, a aspirina reduziu o risco relativo de $\mathrm{TEV}$ recorrente em $32 \%$ e a dose de $20 \mathrm{mg}$ de rivaroxabana reduziu em $82 \%$. Consoante com esses achados, o presente estudo mostrou que, em comparação com a aspirina, tanto o grupo de $20 \mathrm{mg}$ quanto o de $10 \mathrm{mg}$ de rivaroxabana reduziram o risco relativo de recorrência de TEV em $70 \%$.

Os autores concluíram que o risco de recorrência de TEV foi significantemente menor com rivaroxabana nas doses de $20 \mathrm{mg}$ ou $10 \mathrm{mg}$ do que com $100 \mathrm{mg}$ de aspirina sem aumento significante na taxa de sangramento.

\section{REFERÊNCIA}

1. Weitz JI, Lensing AWA, Prins MH, Bauersachs R, BeyerWestendorf J, Bounameaux H, et al. Rivaroxaban or aspirin for extended treatment of venous thromboembolism. N Engl J Med. 2017;376(13):1211-22. http://doi.org/10.1056/ NEJMoa1700518

${ }^{1}$ Pontifícia Universidade Católica de São Paulo, Faculdade de Ciências Médicas e da Saúde - Sorocaba (SP), Brasil. Autora correspondente: Andressa Zacharias Bendine - Pontifícia Universidade Católica de São Paulo, Faculdade de Ciências Médicas e da Saúde - Rua Joubert Wey, 290 - CEP: 18030-070 - Sorocaba (SP), Brasil - E-mail: dre_nos@hotmail.com Recebido em 19/07/2019. Aceito para publicação em 26/08/2019.

Como citar este artigo:

Bendine AZ, Costa JA. Qual droga utilizar para o tratamento prolongado de tromboembolismo venoso? Rev Fac Ciênc Méd Sorocaba. 2020;22(2):85. https://doi.org/10.23925/1984-4840.2020v22i2a9 